\title{
Ciclosporin in psoriasis clinical practice: an international consensus statement
}

\author{
C.E.M.GRIFFITHS, L.DUBERTRET, ${ }^{*}$ C.N.ELLIS $\uparrow \dagger$ A.Y.FINLAY, $\$$ A.F.FINZI, $\S$ \\ V.C.HO, A.JOHNSTON, ** A.KATSAMBAS, $\dagger \dagger$ A-E.LISON, $+\ddagger$ J.M.NAEYAERT, $\S$ \\ H.NAKAGAWA, C.PAUL*** AND F.VANACLOCHA $+\uparrow \dagger$ \\ Dermatology Centre, University of Manchester, Hope Hospital, Manchester, UK \\ *Skin Research Institute and Dermatology, Saint Louis University Hospital, Paris, France \\ $\uparrow$ Department of Dermatology, University of Michigan Medical School, Ann Arbor, Michigan, USA \\ \$Department of Dermatology, University of Wales College of Medicine, Cardiff, UK \\ §Department of Dermatology, University of Milan, Milan, Italy \\ - Division of Dermatology, University of British Columbia, Vancouver, British Columbia, Canada \\ **Clinical Pharmacology, Barts and The London, University of London, UK \\ $\dagger \dagger$ Department of Dermatology, University of Athens, A.Sygros Hospital, Athens, Greece \\ †Department of Nephrology and Transplantation, Medizinische Klinik III, Zentralkrankenhaus, Bremen, Germany \\ $\S \S D e p a r t m e n t$ of Dermatology, Ghent University Hospital, Gent, Belgium \\ - - Department of Dermatology, Jichi Medical School, Tochigi, Japan \\ ***Clinical Research Department, Novartis Pharma AG, Basel, Switzerland, and Department of Dermatology, Mulhouse General \\ Hospital, Mulhouse, France \\ ††'Department of Dermatology, Hospital 12 de Octubre, Madrid, Spain
}

Summary The main recommendations for the use of ciclosporin in the management of psoriasis are: (i) intermittent short courses (average of 12 weeks duration) of ciclosporin are preferable; (ii) ciclosporin should be given in the dose range $2 \cdot 5-5 \cdot 0 \mathrm{mg} \mathrm{kg}^{-1}$ day $^{-1}$ (doses greater than $5.0 \mathrm{mg} \mathrm{kg}{ }^{-1}$ day $^{-1}$ should only be given in exceptional circumstances); (iii) treatment regimens should be tailored to the needs of each patient; (iv) selection of patients should take into account psychosocial disability, as well as clinical extent of disease and failure of previous treatment; (v) each patient's renal function (as measured by serum creatinine) should be thoroughly assessed before and during treatment; (vi) each patient's blood pressure should be carefully monitored before and during treatment; (vii) adherence to treatment guidelines substantially reduces the risk of adverse events; (viii) long-term continuous ciclosporin therapy may be appropriate in a subgroup of patients; however, duration of treatment should be kept below 2 years whenever possible; and (ix) when long-term continuous ciclosporin therapy is necessary, annual evaluation of glomerular filtration rate may be useful to accurately monitor renal function.

Key words: calcineurin inhibitor, ciclosporin, clinical practice, consensus, psoriasis

\footnotetext{
Correspondence: Professor Christopher Griffiths, Dermatology Centre, University of Manchester Irving Building, Hope Hospital, Salford, Manchester, M6 8HD, UK. E-mail: christopher.griffiths@man.ac.uk. This report has been generated from the 'Ciclosporin in Psoriasis Clinical Practice' consensus conference held in Paris, France, on 5 September 2003. It involved experts in dermatology, clinical pharmacology and nephrology. The purpose of the conference was to develop a consensus statement on the clinical role of ciclosporin in the treatment of psoriasis. This conference was supported by an unrestricted educational grant from Novartis Pharma AG.
}

\section{Introduction}

The efficacy of ciclosporin $\left(\mathrm{Neoral}^{\circledR}\right)$ in the treatment of severe plaque psoriasis is unquestionable, as demonstrated by data from clinical trials. ${ }^{1}$ Dermatologists accept this, although many clinicians continue to have concerns regarding its use. These concerns primarily relate to preconceptions surrounding sideeffects, such as renal impairment and hypertension, and the lack of guidance regarding the appropriate and effective use of ciclosporin in the treatment of severe psoriasis. 
An international conference was convened to address these issues, by undertaking a multidisciplinary expert review of the clinical data currently available on ciclosporin therapy in psoriasis. The meeting endeavoured to provide a comprehensive revision of relevant clinical practice since the previous consensus conference in $1996 .^{2}$ The aims of the conference were: (i) to examine the evidence for intermittent, short-course ciclosporin therapy; (ii) to provide evidence-based guidance on the effective use of ciclosporin therapy for the treatment of psoriasis, and on the monitoring of potential side-effects associated with its use; and (iii) to present a unified methodology for the use of ciclosporin in clinical dermatology practice.

The recommendations outlined in this document are proposed only as a guide, and are intended for use in conjunction with the physician's clinical judgement.

\section{Patient selection for ciclosporin therapy in psoriasis}

Patient selection for ciclosporin therapy should take into account clinical extent of disease and/or psychosocial disability, and/or response of severe disease to previous treatment.

On clinical assessment, severe psoriasis is usually defined as disease affecting more than $10-20 \%$ of the skin, or as a Psoriasis Area Severity Index (PASI) $>10$. However, difficult to treat or 'severe' psoriasis does not necessarily equate with clinical extent of disease. ${ }^{3}$ Furthermore, the impact of psoriasis on a patient's quality of life (QoL) is often disproportionate to the clinical severity of the condition. For example, one patient with clinically minimal psoriasis may experience greater psychosocial disability than another with far more extensive disease. Although there is some relationship between severity of disease (as measured by PASI) and QoL, ${ }^{4}$ self-reported severity (rather than PASI) has been found to be the most significant predictor of QoL. ${ }^{5}$

Ensuing loss of self-confidence and concerns over physical appearance can impact on all areas of a patient's life, including personal relationships and employment. Unfortunately, there is frequently a mismatch in perception between patient and dermatologist regarding the impact of skin disease on QoL. ${ }^{6}$ Patients with psoriasis frequently feel highly stigmatized by their condition, which has a profoundly negative effect on QoL. ${ }^{7}$

There is an important relationship between QoL issues and treatment compliance. Patients with poor
QoL are less likely to adhere to their medication, ${ }^{8}$ particularly younger patients and those with clinically severe disease. ${ }^{9}$ QoL may also have an impact on treatment outcome, as psychological distress is known to impair clearance of psoriasis in patients treated with photochemotherapy. ${ }^{10}$

How then should 'severe' disease be defined? Traditional methods have used physical sign-based assessment, however, there has been a recent trend to incorporate psychological evaluation, with particular emphasis on QoL. ${ }^{3,11,12}$ The only remaining problem with the integration of QoL scores into patient assessment/intervention is that there is currently no definition on their practical meaning, in terms of clinical decision-making.

'Severe' disease can also incorporate psoriasis that is of limited extent but is physically disabling, such as on the palms or soles, and disease that has been resistant to other systemic therapies and/or when the patient is unable to tolerate other systemic therapies.

In summary, when considering a patient for ciclosporin therapy, an amended definition of disease severity should be used that includes psychosocial disability, as well as the clinical extent of disease and previous response to treatment.

\section{Clinical efficacy of ciclosporin in psoriasis}

Ciclosporin is proven to rapidly induce remission of psoriasis, significantly reduce the severity and extent of disease, control disease symptoms, and improve quality of life.

It is proven to be among the best therapeutic options for the treatment of 'severe' psoriasis, and a large evidence base supports its use. Although an array of medication is available for the therapy of severe psoriasis, a significant number of patients remains greatly dissatisfied with the efficacy of their treatments. ${ }^{13}$ The limitations of current therapies impact on treatment compliance and, ultimately, on clinical outcome. Consequently, there is a genuine need for a therapy that is effective in achieving disease control and remission, enhances QoL, and is relatively safe and well tolerated; ciclosporin is such a therapeutic agent.

Data from 18 randomised controlled trials (RCTs) examining the efficacy and safety of ciclosporin in the treatment of severe psoriasis are currently available. ${ }^{1}$ Of these, 13 RCTs investigated induction of remission and five concerned maintenance therapy. These studies used either short-course (average of 12 weeks) or longterm ciclosporin therapy regimens. 
The efficacy of intermittent short courses of ciclosporin is confirmed by data from various studies. Ciclosporin at doses of $2 \cdot 5-5 \cdot 0 \mathrm{mg} \mathrm{kg}^{-1}$ day $^{-1}$ given for 12-16 weeks rapidly produced marked improvement or complete clearance of disease in $80-90 \%$ of patients. ${ }^{14-19}$ Data from Faerber et al. ${ }^{19}$ demonstrate the efficacy of ciclosporin in the dose range 2.5$5 \cdot 0 \mathrm{mg} \mathrm{kg}^{-1}$ day $^{-1}$ (Fig. 1). The Psoriasis Intermittent Short Course of Efficacy of Sandimmun Neoral (PISCES) study involved 400 patients with severe psoriasis who were given up to four courses of ciclosporin (2.5-5.0 mg kg${ }^{-1}$ day $^{-1}$ for a maximum of 12 weeks). ${ }^{17}$ Results from PISCES demonstrated that in some patients sustained remission could be achieved after only one course of ciclosporin therapy in that $45 \%$ of subjects had not relapsed 4 months after stopping treatment and $31 \%$ had not relapsed after 6 months. Significant improvements in QoL (91\%), disease symptoms (98\%), and PASI (91\%) were also recorded with intermittent short-course therapy. ${ }^{20}$

Longer-term, continuous ciclosporin therapy may be required to maintain disease remission in a small

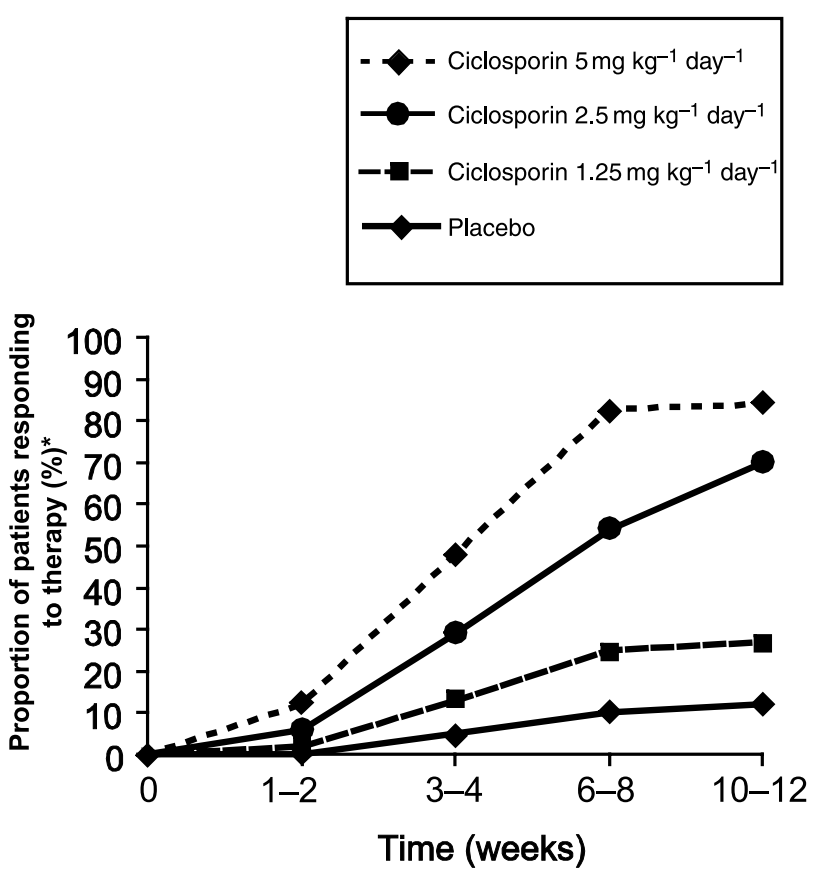

Figure 1. Ciclosporin short-course therapy: response rate. Ciclosporin $2.5-5.0 \mathrm{mg} \mathrm{kg}^{-1} \mathrm{day}^{-1}$ is effective in a high proportion of patients, as shown by data from a meta-analysis of 510 patients with severe plaque psoriasis treated with ciclosporin or placebo (total of 756 treatment cycles, maximum duration 12 weeks). *Proportion of patients with $\geq 50 \%$ reduction in psoriasis area and PASI score over the 12-week course of treatment. (NB: recommended starting dose of ciclosporin is $2.5-5.0 \mathrm{mg}^{-1} \mathrm{~kg}$ day $\left.^{-1}\right) .{ }^{19}$ proportion of patients suffering from recalcitrant psoriasis. Mean study doses of ciclosporin of $3 \cdot 0-3 \cdot 5 \mathrm{mg} \mathrm{kg}^{-1} \mathrm{day}^{-1}$ were given for varying periods of time, ranging from $6^{21,22}$ through $17^{23}$ and up to 56 months. $^{24}$ Significant clinical improvement was observed in $86^{21}$ to $95 \%{ }^{23}$ of patients, and the majority $\left(58^{21}\right.$ to $\left.91 \%^{22}\right)$ was maintained on a ciclosporin dose $<3.5 \mathrm{mg} \mathrm{kg}^{-1}$ day $^{-1}$. During a long-term study in which patients were treated for at least 12 months with three doses of ciclosporin $(1 \cdot 25,2.5$ and $5.0 \mathrm{mg} \mathrm{kg}^{-1} \mathrm{day}^{-1}$, respectively), $12 \cdot 5 \%$ were maintained on the lowest dose $\left(1.25 \mathrm{mg} \mathrm{kg}^{-1} \mathrm{day}^{-1}\right)$ without loss of efficacy. ${ }^{25}$ From this it may be concluded that ciclosporin doses $<2.5 \mathrm{mg} \mathrm{kg}^{-1}$ day $^{-1}$ may be sufficient to induce a remission in a subgroup of patients, which makes individual dose-adjustment mandatory. QoL scores also improved markedly following 12 months of ciclosporin treatment, with patients' perceptions of good health rising from $18 \%$ to $67 \%$ and rates of psychiatric morbidity falling from $69 \%$ to $32 \%{ }^{26,27}$

In summary, data from RCTs have established ciclosporin as an agent rapidly effective in achieving control of psoriasis and inducing remission. Ciclosporin also considerably improves patient QoL.

\section{Management of renal side-effects}

Renal side-effects associated with ciclosporin are dose-related and occur almost exclusively during prolonged exposure to ciclosporin and/or at doses $>5.0 \mathrm{mg} \mathrm{kg}^{-1} \mathrm{day}^{-1} \cdot{ }^{28,29}$

Ciclosporin therapy causes increased vascular resistance, which may result in reduced renal plasma flow and decreased clearance of endogenous creatinine. Subsequently this can manifest as an increase in serum creatinine. Current treatment guidelines stipulate reduction of ciclosporin dose if serum creatinine increases by $30 \%$ above the baseline value (even if this increase is within the normal range). ${ }^{30,31}$ However, any changes in renal performance are usually functional and are normally quickly reversed by cessation of ciclosporin therapy (if required). ${ }^{29}$

During continuous treatment with ciclosporin, the proportion of patients experiencing an elevation in serum creatinine above the baseline value increases over time. Thus, only a minority of patients can be maintained on ciclosporin for five continuous years or more without experiencing changes in renal function. $^{32,33}$ Study data have revealed that ciclosporin nephropathy is intimately related to drug dose 
(i.e. $>5 \cdot 0 \mathrm{mg} \mathrm{kg}^{-1} \mathrm{day}^{-1}$ ) and duration of treatment. $^{28,29,34}$ The risk of renal toxicity from ciclosporin during treatment of psoriasis is reduced by the use of intermittent, short courses of the drug. Intermittent therapy provides 'drug holidays' to allow renal recovery and restoration of normal renal function, thereby returning serum creatinine concentration to baseline levels. ${ }^{28}$ Renal safety during short-course ciclosporin therapy is verified by the fact that only a minority of patients $\left(4 \%,{ }^{19} 17 \%{ }^{16}\right.$ and $\left.10-27 \%{ }^{17,18}\right)$ experienced an elevation in serum creatinine, which was typically transient and commonly returned to baseline within 4 weeks following dose reduction or treatment cessation. ${ }^{17}$

The chance of developing renal impairment during ciclosporin therapy can be minimized by screening patients at the baseline assessment for any risk factors of renal toxicity. These include pre-existing or new-onset hypertension, advanced age, renal inflammatory conditions, and abnormalities in absorption of ciclosporin. In patients with psoriasis, concomitant medication, age, weight, and the presence of hypertension are also important risk factors for potential ciclosporin nephropathy. ${ }^{31}$ Most importantly, the dose of ciclosporin used should only exceed $5.0 \mathrm{mg} \mathrm{kg}^{-1} \mathrm{day}^{-1}$ in exceptional circumstances and the duration of treatment for each patient should be only as long as is necessary to achieve clearance (or near-clearance) of disease. Extended periods of continuous ciclosporin use should be avoided wherever possible, as changes in renal histology have been reported after 3 years of such therapy. ${ }^{35}$

In summary, ciclosporin therapy is associated with a risk of renal toxicity that is related to both dose of drug and duration of treatment. As long as each patient is properly screened, appropriately dosed, and monitored regularly, risk can be controlled and corrected. Further management guidelines for monitoring renal safety during ciclosporin therapy in psoriasis can be found on page 18 of this supplement.

\section{Risk of malignancy during use of ciclosporin in psoriasis}

\footnotetext{
Non-melanoma skin cancer

Psoriasis patients treated with ciclosporin have a significantly higher risk of non-melanoma skin cancer (NMSC) compared with the non-psoriatic population (relative risk $6: 1){ }^{36}$ However, this increased risk is observed exclusively in patients who have been exposed previously to psoralen-ultraviolet A (PUVA) therapy. ${ }^{36,37}$
}

The long-term use of immunosuppressants such as ciclosporin is associated with a potential increase in the risk of developing certain types of malignant tumour, specifically cancers of the skin and lymphoid tissue. ${ }^{38,39}$ A recent prospective cohort study investigated the incidence of malignancy in patients with severe psoriasis, who were on long-term therapy with ciclosporin $(n=1252$; mean length of treatment 1.9 years; mean dose $2 \cdot 7-3 \cdot 1 \mathrm{mg} \mathrm{kg}^{-1}$ day $^{-1}$ ). ${ }^{36}$ Data showed the overall incidence of malignancies in the study cohort was twice that of the general population. This was attributed to the six-fold higher incidence of skin cancers, the majority of which were squamous cell carcinomas (SCC).

However, the risk of malignancy, particularly of the skin, is higher in people with psoriasis than in the general population in any case, ${ }^{36,40-42}$ and exposure to certain treatments for severe psoriasis are significant risk factors for NMSC. For example, PUVA is associated with a higher risk of NMSC, and this may be affected by immunosuppressants (see below). Multivariate analysis of the ciclosporin patient cohort in the study of Paul et $a{ }^{36}{ }^{36}$ indicates that exposure to PUVA, methotrexate, and other immunosuppressants are all significant risk factors for NMSC (relative risks of $7 \cdot 3,4.6$ and $3 \cdot 5$, respectively). Patients with prolonged exposure to ciclosporin ( $>2$ years cumulative treatment) had a significantly increased risk of NMSC than those with shorter exposures ${ }^{36}$ (Table 1).

PUVA is a tumour initiator that causes mutation in various oncogenes ( $p 53, \mathrm{H}$-ras). ${ }^{43,44}$ The risk of SCC increases linearly with the number of PUVA sessions received; the incidence of SCC is 14 -fold greater for a patient who has received more than 200 PUVA treatments compared with one who has received fewer than 100 treatments. $^{43,45}$ This risk is even larger for patients with skin phototypes I and II, or with previous high exposure to tar/UVB. In the ciclosporin cohort study, all patients who developed

Table 1. Standardized incidence ratio by malignancy and exposure to ciclosporin $^{36}$

\begin{tabular}{lcl}
\hline & SIR $^{*}$ & $95 \% \mathrm{CI}^{*}$ \\
\hline Any malignancy & 2.1 & $1.6-2.9$ \\
Any skin malignancy & 6.1 & $3.8-9.1$ \\
Non-melanoma skin malignancy & 6.2 & $3.8-9.5$ \\
Malignant melanoma & 4.7 & $0.6-17.0$ \\
Any non-skin malignancy & 1.3 & $0.8-1.9$ \\
\hline
\end{tabular}

* Compared with normal population. SIR, standardized incidence ratio; $\mathrm{CI}$, confidence interval. 
SCC had received PUVA before the initiation of ciclosporin. $^{36}$

Suggested management guidelines include the following; (i) narrow-band UVB therapy, rather than PUVA, should be the preferred first-line agent; (ii) where PUVA is used, treatments should be limited to fewer than 200 sessions $\left(1000 \mathrm{~J} \mathrm{~cm}^{-2}\right.$ ); (iii) the use of immunosuppressant therapy in combination with UV phototherapy is not recommended; (iv) during rotational therapy, the use of ciclosporin should be avoided immediately prior to and especially following photo (chemo)therapy (PUVA); (v) exposure to immunosuppressants should be limited (intermittent shortcourse therapy is preferred for ciclosporin) in patients with a high cumulative exposure to PUVA; (vi) patients with skin phototypes I and II should be closely monitored; and (vii) patients with a previous history of skin cancer should also be closely monitored, and the use of immunosuppressants avoided in individuals who have had melanoma or SCC. However, the use of methotrexate, retinoids and fumarates before/after ciclosporin therapy is permitted, and oral retinoids may be beneficial in controlling PUVA/ciclosporininduced premalignant/malignant lesions. ${ }^{46}$

If one considers that a patient may require ciclosporin treatment at some point in the future, it may be appropriate to initiate treatment before PUVA in the therapy cycle.

\section{Non-skin malignancies}

There is no significant increase in risk of non-skin cancer with ciclosporin therapy compared with the general population. ${ }^{36}$

Paul et $a l .{ }^{36}$ found the incidence of non-skin cancer in the psoriasis patient cohort was not significantly different to that observed in the general population. Prolonged exposure to ciclosporin ( $>2$ years cumulative treatment) was not associated with a higher risk of non-skin cancer. Multivariate analysis shows that exposure to PUVA was the only significant risk factor for non-skin malignancies (relative risk 2.5). However, this cohort was too small to provide meaningful results for individual types of non-skin cancers.

In summary, the treatment of psoriasis with ciclosporin is associated with an increased incidence of NMSC in those patients previously exposed to PUVA. The risk of non-skin malignancies does not appear to be increased in psoriasis patients treated with ciclosporin; however, larger studies are needed to confirm these findings.

\section{Clinical considerations for ciclosporin therapy in psoriasis}

Patient management protocols have been established for the use of ciclosporin in psoriasis, making drug initiation and administration simple and straightforward.

A summary of points to consider during the use of ciclosporin in the treatment of psoriasis and recommendations for clinical practice can be found in Table 2. The fundamental point is that therapy should always be tailored to the needs of the individual patient.

The efficacy of ciclosporin has been discussed above; however, this agent also possesses several side-effects that must be considered prior to the commencement of therapy, the most important of these being renal dysfunction and hypertension. Other side-effects include gastrointestinal symptoms, hypertrichosis, gingival hyperplasia, musculoskeletal symptoms (joint pain, leg cramps), neurological symptoms (headache, tremor, paraesthesia), fatigue and metabolic abnormalities (hyperbilirubinaemia, hypercalcaemia, hypomagnesaemia, hyperuricaemia, hypertriglyceridaemia and hypercholesterolaemia). If the presence of any of these causes concern, the clinician should initiate dose reduction and/or cessation of ciclosporin until the side-effect diminishes. In the event of gingival hyperplasia, which is usually associated with poor oral hygiene, the patient should consult a dentist.

The clinician should be aware of any drugs that have the potential to either increase or decrease the systemic exposure to ciclosporin when administered concomitantly. ${ }^{47}$ There are three main types of drug interaction: (i) drugs that interfere with ciclosporin bioavailability and/or metabolism (such as inducers or inhibitors of cytochrome $\mathrm{P}_{450}$ iso-enzymes); (ii) drugs that have potentially nephrotoxic effects; and (iii) drugs whose metabolism may be affected by ciclosporin (Table 3).

Baseline blood pressure (BP) should be assessed carefully to screen for hypertension (defined as $\geq 140 / 90 \mathrm{mmHg}$ ). Correct BP measurement technique is vital and national hypertension society websites should be consulted for guidance. Guidelines from the World Health Organization, ${ }^{48}$ European Society of Hypertension, ${ }^{49}$ and/or US Joint National Committee on Hypertension ${ }^{50}$ should be used as reference for the definition and management of arterial hypertension. Blood pressure should be measured at 2-weekly intervals for the first 2 months of ciclosporin therapy, and monthly thereafter. If $\mathrm{BP}$ is noted to increase beyond the upper limits of normal (i.e. systolic 
Table 2. Ciclosporin in psoriasis: clinical practice guidelines

\begin{tabular}{|c|c|}
\hline Clinical category & Details \& recommendations \\
\hline Patient selection; points to consider & $\begin{array}{l}\text { Patient's perception of disease severity } \\
\text { QoL impairment } \\
\text { Psychiatric morbidity } \\
\text { Patient's willingness to accept intermittent relapse following treatment cessation } \\
\text { 'Objective' measurements of disease severity } \\
\text { Clinical extent of disease } \\
\text { Failure of previous treatment(s) } \\
\text { Efficacy \& side effects of potential treatment }\end{array}$ \\
\hline Indications for particular caution in use & $\begin{array}{l}\text { Malignancy / pre-malignant conditions: current or previous; excluding basal cell carcinoma } \\
\text { Immunodeficiency disorders: primary or secondary } \\
\text { Abnormal renal function } \\
\text { Severe hepatic dysfunction; hepatitis C } \\
\text { Hypertension* (controlled or uncontrolled): pre-existing or new onset } \\
\text { Severe infection: of any type } \\
\text { Unable or unwilling to undergo regular monitoring } \\
\text { Diabetes* } \\
\text { Obesity (dose ciclosporin at patient's ideal body weight) } \\
\text { Aged > } 65 \text { years* } \\
\text { Current/previous photo(chemo)therapy } \\
\text { Drug/ alcohol abuse } \\
\text { (Note: Pregnancy is not known to be an absolute contra-indication to the use of ciclosporin) }\end{array}$ \\
\hline Pre-treatment assessment & $\begin{array}{l}\text { Complete medical history \& full physical/dermatological examination } \\
\text { Risk factors for renal toxicity } \\
\text { Renal inflammation, abnormal ciclosporin absorption, (see also * above) } \\
\text { Baseline renal function } \\
\text { Serum creatinine (taken after 12-hour fast, on at least } 2 \text { occasions) } \\
\text { Baseline blood pressure (taken using proper technique, on at least } 2 \text { occasions, should } \\
\text { be }<140 / 90 \mathrm{mmHg}) \dagger \\
\text { Malignancy screening } \\
\text { Patients should be advised to follow national recommendations for cancer screening } \\
\text { (cervix, breast \& prostate) } \\
\text { Other investigations } \\
\text { Liver function tests \& serum } \mathrm{K}^{+} \text {should be measured } \\
\text { Serum lipids, uric acid, bilirubin \& } \mathrm{Mg}^{2+} \text { are also commonly assessed }\end{array}$ \\
\hline Starting dose \& treatment regimen & $\begin{array}{l}\text { Dose } \\
2.5-5.0 \mathrm{mg} \mathrm{kg}^{-1} \text { day }^{-1} \text { (usually given as } 2 \text { divided doses) } \\
\text { Begin at the lower range \& titrate according to clinical response } \\
\text { Regimen } \\
\text { Intermittent short-course therapy is preferred } \\
\text { Aim for disease clearance within } \sim 12 \text { weeks } \\
\text { It is not necessary to taper off the dose at the end of treatment } \\
\text { Keep duration of treatment to a minimum } \\
\text { Treatment should be tailored to the needs of each patient }\end{array}$ \\
\hline Monitoring during treatment & $\begin{array}{l}\text { Blood pressure } \\
\text { Measure at }+2 \text { weeks, }+4 \text { weeks } \&+6 \text { weeks, then measure monthly thereafter } \$ \\
\text { Renal function } \\
\text { Measure serum creatinine at } 2 \text {-weekly intervals for the first } 2 \text { months, then measure monthly } \\
\text { thereafter } \\
\text { For patients on long-term therapy ( }>1 \text { year continuous treatment) assess annual renal } \\
\text { function using creatinine clearance to measure GFR } \S \\
\text { Serum lipids } \& \mathrm{Mg}^{2+} \text { should be measured } 6 \text { monthly }\end{array}$ \\
\hline
\end{tabular}

$\S$ See also Fig. 4. GFR, glomerular filtration rate; $\mathrm{K}^{+}$, potassium; $\mathrm{Mg}^{2+}$, magnesium; QoL, quality of life. $†$ See WHO guidelines, ${ }^{48}$ ESH guidelines $^{49}$ or JNC-VII ${ }^{50}$ for current definition of hypertension. See also National Hypertension Society websites for guidance on BP recording, e.g. BHS website (UK) http://w3.abdn.ac.uk/BHS. $\ddagger$ More frequent readings will be necessary if values become abnormal.

BP > $139 \mathrm{mmHg}$; diastolic $\mathrm{BP}>89 \mathrm{mmHg})$, the protocol outlined in Fig. 2 should be followed. Rising $\mathrm{BP}$ should be controlled using clinically appropriate methods, such as reduction in ciclosporin dose of $25-50 \%$ and/or the addition of an appropriate antihypertensive agent. If dihydropyridine-type calcium 
Table 3. Potential drug interactions with ciclosporin therapy

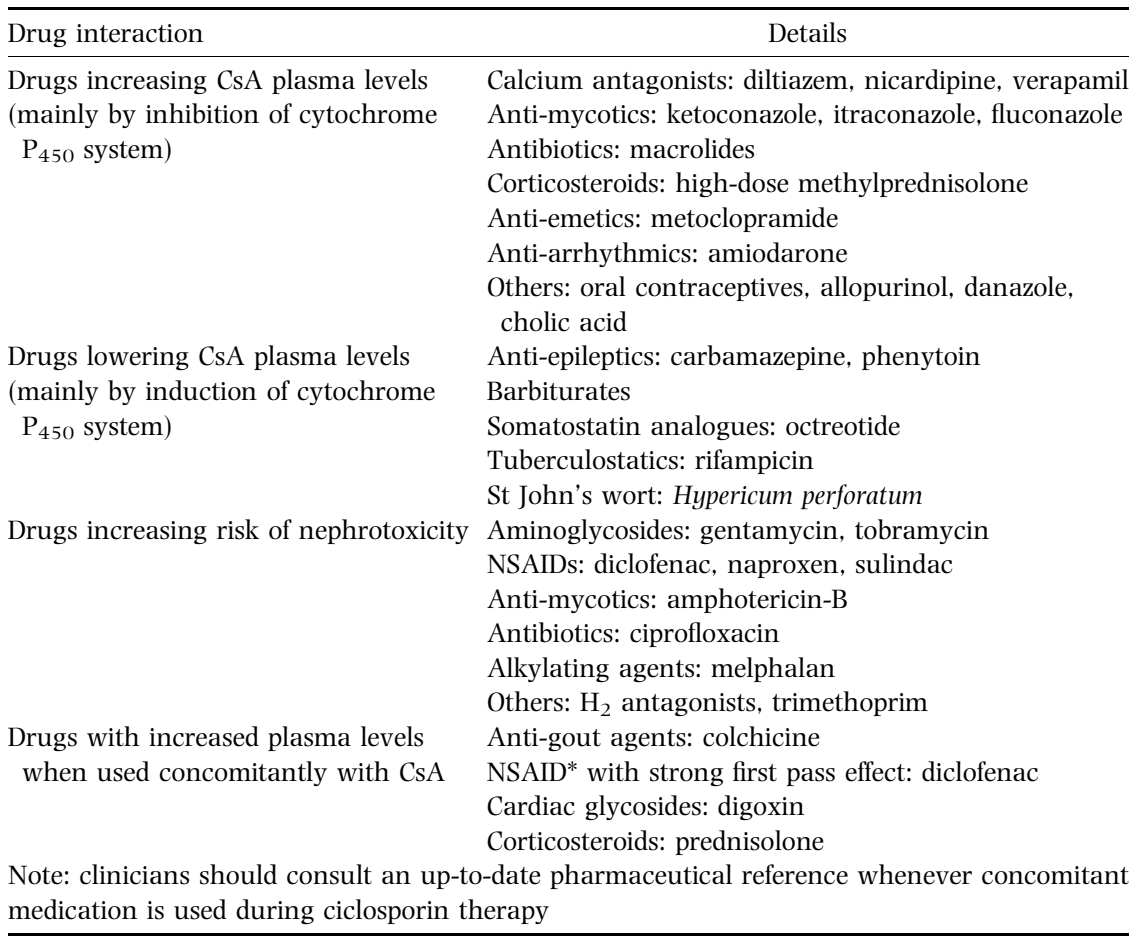

CsA, ciclosporin; NSAID, non-steroidal anti-inflammatory drug. * Salicylic acid can be used. channel blockers are selected, nifedipine should not be used due to the potential for a synergistic action with ciclosporin in the development of gingival hyperplasia. Data from RCTs have shown that only a minority of patients $(\sim 5-12 \%)$ developed new-onset hypertension during short-course ciclosporin therapy, and the overwhelming majority of these cases responded to dose reduction and/or antihypertensive medication. ${ }^{17,19}$

Screening for renal toxicity caused by ciclosporin therapy has been discussed above. Serum creatinine should be measured, on at least two separate occasions, following a 12-h fast. Patients should also be advised to refrain from strenuous exercise immediately prior to testing, as muscle metabolism can elevate serum creatinine. Serum levels of creatinine should be measured at 2-weekly intervals for the first 2 months of ciclosporin therapy, and monthly thereafter. If serum creatinine is noted to increase by $30 \%$ or more of the value at baseline (even if this figure remains within the normal range), the protocol outlined in Fig. 3 should be followed. Ciclosporin nephropathy usually responds rapidly $(<4$ weeks) to either reduction in dose or cessation of ciclosporin treatment. ${ }^{17}$

It should be noted that a small number of patients may have normal serum levels of creatinine but a diminished glomerular filtration rate (GFR), which indicates the presence of renal dysfunction. Measurement of GFR is not routinely carried out in clinical dermatology practice. However, creatinine clearance can be easily calculated from the serum creatinine value using the Cockcroft-Gault equation, which provides a good estimate of GFR (Fig 4). As ciclosporin nephropathy is closely related to duration of therapy, GFR should be assessed annually in patients receiving long-term continuous ciclosporin treatment. ${ }^{33}$

Patients should be advised to follow national recommendations for cancer screening as appropriate (for women, cervical smear, breast examination/imaging, etc.; for men, prostate cancer screening). Recommendations for the management of patients at risk of/with a previous history of skin cancer are discussed above.

If a patient contracts an infection while receiving ciclosporin therapy, the clinical response will depend on the type of infectious agent and the severity of the infection. For herpes virus infections, ciclosporin therapy may be continued if the signs/symptoms are mild and/or controlled by oral antivirals. For bacterial or fungal infections, the patient should be treated promptly with an appropriate antibiotic/antimycotic agent (avoiding systemic macrolides and azoles, as these drugs inhibit $\mathrm{P}_{450}$ iso-enzymes and elevate blood ciclosporin levels). Clinicians should also be aware that 


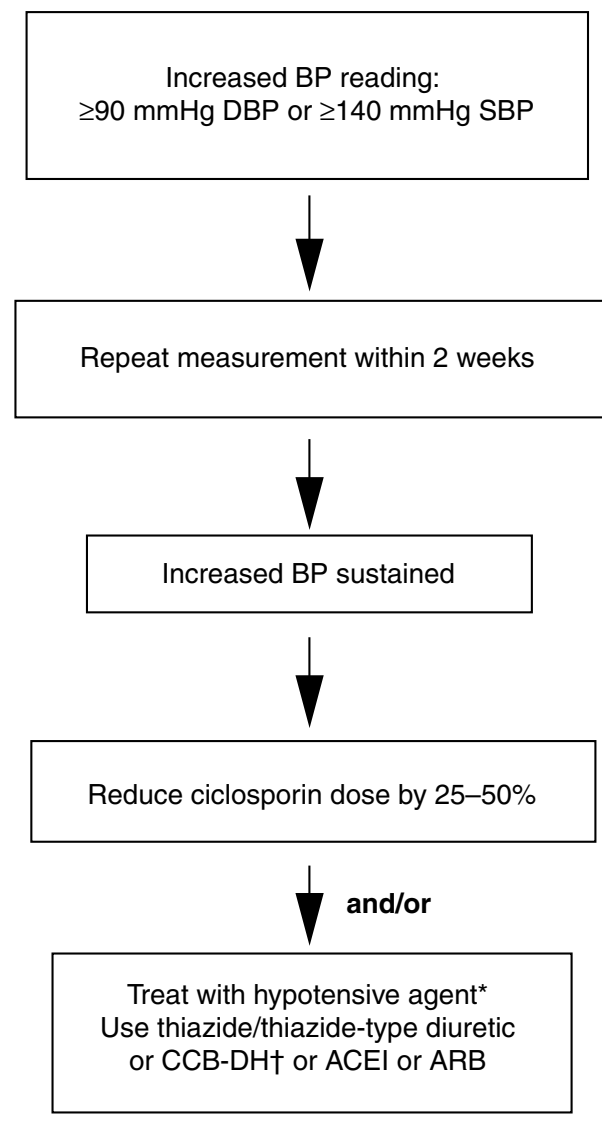

Figure 2. Rising blood pressure during ciclosporin therapy: management. ${ }^{*}$ The major guidelines ${ }^{48-50}$ recommend that initial therapy for uncomplicated hypertension should begin with a low-dose thiazide or thiazide-type diuretic, long-acting dihydropyridine-CCB, ACEI or ARB. If there are any adverse effects with the initial choice of agent, one of the other drug classes mentioned should then be substituted. $\uparrow$ Nifedipine should be avoided as it acts synergistically with ciclosporin to cause gingival hyperplasia. ACEI, angiotensin converting enzyme inhibitor; ARB, angiotensin II receptor blocker; $\mathrm{BP}$, blood pressure; CCB-DH, calcium channel blocker-dihydropyridine type; DBP, diastolic BP; SBP, systolic BP.

vaccination may be less effective during ciclosporin therapy, and the use of live attenuated vaccines should be avoided.

There is still debate as to whether routine monitoring of ciclosporin blood levels is necessary. In principle, two methods of measurement are available. Trough-level monitoring $\left(\mathrm{C}_{0}\right)$ is common but does not necessarily reflect ciclosporin exposure as a function of drug intake. This is better assessed by measuring the concentration of ciclosporin $2 \mathrm{~h}$ after drug intake $\left(\mathrm{C}_{2}\right) .{ }^{51}$ Unlike in transplantation medicine, ciclosporin trough blood levels do not properly predict renal dysfunction and do not correlate with clinical outcome. ${ }^{53} \mathrm{~A}$ recent literature review concluded that

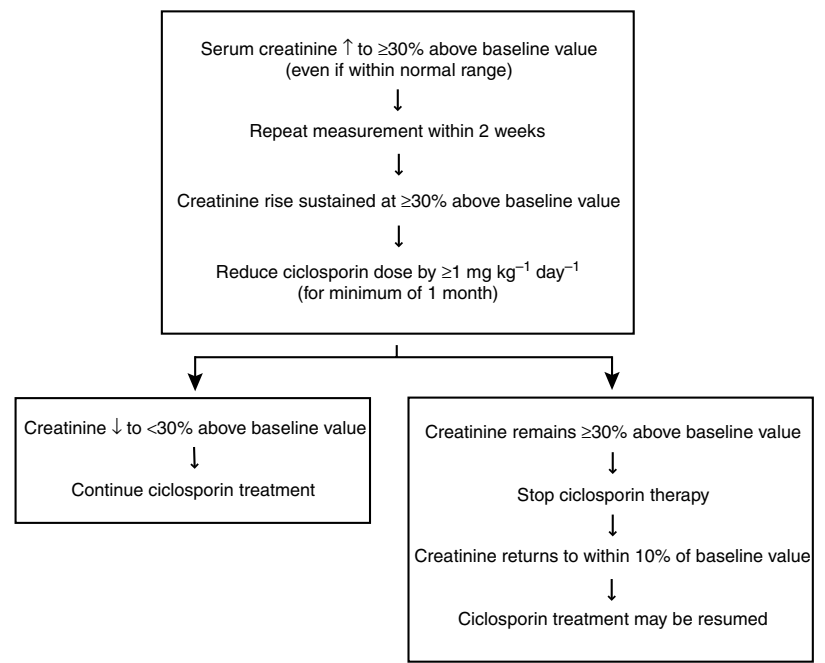

Figure 3. Renal toxicity during ciclosporin therapy: management.

$$
\begin{aligned}
& \text { Men: Creatinine clearance }(\mathrm{mL} / \mathrm{min})=\frac{(140-\text { age }[\text { years] }) \times \text { weight }[\mathrm{kg}]}{72 \times \text { serum creatinine }[\mathrm{mg} / \mathrm{dL}]} \\
& \text { Women: Creatinine clearance }(\mathrm{mL} / \mathrm{min})=\frac{(140-\text { age }[\text { years] }) \times \text { weight }[\mathrm{kg}]}{72 \times \text { serum creatinine }[\mathrm{mg} / \mathrm{dL}]} \times 0.85
\end{aligned}
$$

Figure 4. Calculation of glomerular filtration rate via serum creatinine. Creatinine clearance provides a good estimate of glomerular filtration rate (GFR). Creatinine clearance can be calculated via the Cockcroft-Gault equation using serum creatinine values, as shown above. $^{52}$ Alternatively, on-line 'GFR calculators' such as http:// www.nephron.com/mdrd may be used.

trough-level monitoring was not recommended when ciclosporin was used in doses $<3.0 \mathrm{mg} \mathrm{kg}^{-1}$ day $^{-1}$ during short-term treatment. However, at higher doses, $\mathrm{C}_{0}$ or $\mathrm{C}_{2}$ monitoring may be useful in certain patients in order to detect ciclosporin levels above the recommended range. ${ }^{54}$

\section{Prescribing guidelines for ciclosporin therapy in psoriasis}

Many patients with severe psoriasis can be managed with intermittent short-course ciclosporin therapy.

Data from RCTs have shown that short courses of ciclosporin in the range $2 \cdot 5-5 \cdot 0 \mathrm{mg} \mathrm{kg}^{-1} \mathrm{day}^{-1}$ provide optimum efficacy and safety for the induction of disease remission in clinically severe patients. As most patients do well on low doses of ciclosporin, $2.5 \mathrm{mg} \mathrm{kg}^{-1} \mathrm{day}^{-1}$ is the recommended starting dose. This dose can be increased by $0.5-1 \cdot 0 \mathrm{mg} \mathrm{kg}^{-1}$ day $^{-1}$ at intervals of $2-$ 4 weeks, according to clinical response. Treatment with 
ciclosporin is given for an average of 12 weeks, during which time the aim is for a substantial improvement in disease that satisfies both physician and patient. The use of adjunctive topical therapy is encouraged to reduce ciclosporin dose requirements. Once remission has been achieved, ciclosporin therapy is stopped and the patient is monitored for signs of relapse. Upon relapse, ciclosporin treatment should be recommenced at the previous effective dose and titrated as required (to a maximum of $\left.5 \cdot 0 \mathrm{mg} \mathrm{kg}^{-1} \mathrm{day}^{-1}\right)$.

In situations where control of psoriasis needs to be achieved swiftly, such as crisis intervention during acute flares of disease, a so-called 'step-down' dosing approach may be more appropriate. Here, treatment begins at a higher dose (still not exceeding $5 \cdot 0 \mathrm{mg} \mathrm{kg}^{-1} \mathrm{day}^{-1}$ ) and is titrated down as symptoms abate.

Data from the PISCES study confirm the efficacy of the short-course regimen; by day 84 of treatment, approximately $80 \%$ of patients with severe psoriasis had achieved at least 75\% improvement in disease area after the first treatment period ${ }^{17}$ (Fig. 5). Furthermore, a considerable proportion of patients remained in remission for up to 6 months after only one course of treatment. The overwhelming majority of patients $(\sim 80 \%)$ needed only one or two courses of ciclosporin treatment during the 1-year study period. The mean dose requirement was $3 \cdot 0-3.5 \mathrm{mg} \mathrm{kg}^{-1}$ day $^{-1}$ for treatment courses 1 and 2 , and $3 \cdot 5-4 \cdot 0 \mathrm{mg} \mathrm{kg}^{-1}$ day $^{-1}$ for courses 3 and $4 .{ }^{17}$ Median time to relapse was 109 days after course 1 , falling to 52-77 days after courses 2-4. Earlier relapse was found to correlate with previous phototherapy and previous systemic treatment, which may indicate a resistant/active form of disease in some patients. ${ }^{17}$

PISCES safety data demonstrate that only $10 \%$ of patients experienced elevated serum creatinine levels (defined as $\geq 30 \%$ increase above baseline) during the

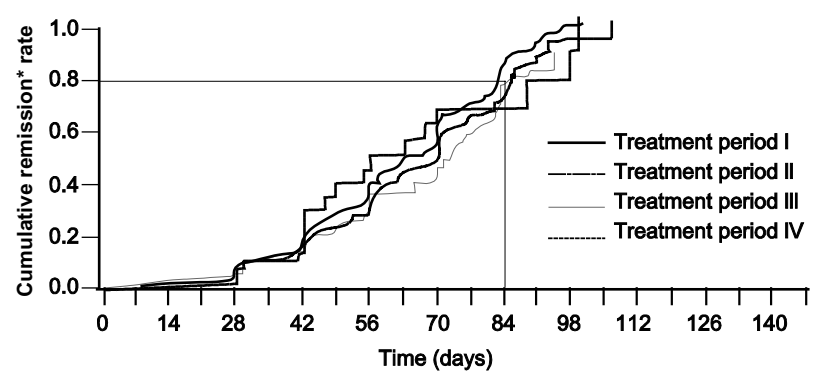

Figure 5. Cumulative rate of treatment success. Data from PISCES-1 study, during which 400 patients with severe psoriasis were treated with short courses of ciclosporin (maximum 12 weeks duration, maximum of four courses of therapy). ${ }^{17} *$ Remission is defined as $75 \%$ improvement in psoriasis disease area. first treatment course, rising to $27 \%$ of patients during the fourth course. ${ }^{17}$ Moreover, the ciclosporin-free periods between treatments allowed renal function to return to normal in affected patients. The safety of short-course ciclosporin therapy was also proven with regard to hypertension, as $\mathrm{BP}$ remained stable in around $85 \%$ of PISCES patients. ${ }^{17}$ The minority that developed new-onset hypertension responded well to either dose reduction and/or cessation of ciclosporin.

A further advantage of short-course ciclosporin therapy is the reduction in duration of exposure to the drug, which consequently lowers the risk of sideeffects. For example, a PISCES extension study found that patients received ciclosporin for only approximately 4 out of every 10 months when using this regimen. $^{18}$

In summary, ciclosporin short-course therapy, when started at a dose of $2 \cdot 5-5 \cdot 0 \mathrm{mg} \mathrm{kg}^{-1}$ day $^{-1}$, offers rapid and significant resolution of extent and severity of disease, sustained remission from psoriasis, positive impact on QoL, reduction in overall exposure time compared to continuous therapy, and has no significant impact on renal function or hypertension when therapeutic guidelines are met.

Longer-term use of ciclosporin as maintenance therapy is indicated in a minority of patients with recalcitrant disease.

A minority of patients has been identified in whom continuous ciclosporin therapy is necessary to maintain disease remission. ${ }^{17,21,23}$ In these patients, the minimum effective dose of ciclosporin required to achieve substantial improvement in disease severity should be used. This dose should be adjusted to provide maximum clinical benefit and minimal drug side-effects, and should not exceed $5 \cdot 0 \mathrm{mg} \mathrm{kg}^{-1} \mathrm{day}^{-1}$. In the majority of studies, patients with refractory disease were maintained on doses $<3.5 \mathrm{mg} \mathrm{kg}^{-1}$ day $^{-1} .^{21-23}$ Continuous treatment duration should be limited to 2 years or less, wherever possible. It is recommended that GFR be measured annually in all patients receiving long-term therapy, owing to the increased risk of ciclosporin-induced nephropathy.

As with short-course treatment, longer-term use of ciclosporin as maintenance therapy offers rapid and sustained remission from disease, and long-term acceptability to many patients. ${ }^{21-23}$ Side-effects are manageable and reversible when therapeutic guidelines are followed. ${ }^{21-23}$ 


\section{Combination and rotational therapy for psoriasis using} ciclosporin.

Several additional ciclosporin treatment regimens have been published, which include combination and rotational therapy concepts. A study by Clark et al. ${ }^{55}$ combined ciclosporin with low-dose methotrexate for use in patients with recalcitrant disease. Rotational therapy using mycophenolate mofetil with long-term ciclosporin therapy was found to be reasonably successful in a study by Davidson et al. ${ }^{56}$ Several other agents have been added to rotational therapy cycles with ciclosporin, including fumarates, sulphasalazine and biologicals. The latter group may offer an alternative to maintenance treatment after induction of remission by short-course ciclosporin. It has been shown that alefacept, a fusion protein of lymphocyte function-associated antigen-3 (LFA-3) and IgG1, although being of lesser clinical efficacy than ciclosporin, can induce a lasting remission in some patients even after cessation of treatment. ${ }^{57}$ Furthermore, efalizumab (an anti-CD11a humanized monoclonal antibody), or etanercept (a tumour necrosis factor$\alpha$-binding fusion protein) may be suitable for maintenance therapy after initial ciclosporin intervention. ${ }^{58,59}$ However, long-term data about the safety of biologicals in psoriasis are still lacking.

The impact on a psoriasis patient of inadvertently switching between alternative formulations of ciclosporin is not fully understood. To minimize any potential risk, it is important that local guidelines are followed to ensure that the appropriate brand is consistently dispensed to the patient.

Ciclosporin is a 'critical-dose' drug, that is, a drug in which a small change in dose or plasma concentration may result in a clinically significant change in efficacy and/or toxicity. Ciclosporin has formulation-dependent bioavailability with a wide interindividual variability in ciclosporin absorption, which explains why individual dosing is required. The Neoral ${ }^{\circledR}$ formulation of ciclosporin offers a far greater consistency in drug delivery than the original Sandimmun ${ }^{\circledR}$ formulation. Recently, generic manufacturers have marketed new formulations of ciclosporin and it is realistic to expect that additional generic formulations will be marketed in the future. However, recommendations from several national bodies state that the prescribing physician should specify the exact brand to be dispensed. This is because there are absorption differences among the assorted formulations of ciclosporin related to pharma- cokinetics, and there is also variation in the way each patient reacts to a particular drug formulation.

Two important concepts are 'bioavailability' and 'bioequivalence'. Bioavailability refers to the rate and extent to which a drug is absorbed from a pharmaceutical formulation and delivered into the general circulation. Two medicinal products are said to be bioequivalent if their bioavailabilities (after administration at the same dose) are similar to such a degree that their effects (i.e. efficacy and safety) are presumed to be essentially the same. Current regulations for demonstrating bioequivalence require only a single positive study, usually a single-dose comparison using healthy male volunteers. The standard pharmacokinetic parameters are examined, and if the mean bioavailability of the comparator drug lies within \pm $20 \%$ of those from the innovator drug, the two drugs are said to be bioequivalent. As there is no requirement to use patients with the target disease; to use women, elderly people, or individuals from different ethnic populations; to use different dose levels of the drugs; or to examine food interactions, the intrinsic limitations of these studies are evident. ${ }^{60-62}$ As these studies only describe the response of a population, they do not address what might happen in an individual patient. Population bioequivalence is adequate to determine if a particular product is of sufficient quality to be prescribed, but gives no indication of an individual's likely reaction to that formulation. For example, a patient who absorbs one ciclosporin formulation satisfactorily will not necessarily absorb another as well, thereby reducing efficacy. Alternatively, that individual may absorb a greater amount, potentially leading to toxicity.

This concept is particularly important for clinicians, who must consider all the possible effects of substituting one brand of ciclosporin for another, principally those pertaining to patient safety and variation in disease control. In line with national recommendations, ${ }^{63}$ prescribing by brand is recommended in order to avoid potential adverse events of unplanned switching. Patients should also be educated about generic drug substitution, so that they are aware if a different formulation is unintentionally obtained.

\section{Conclusions}

The evidence base for ciclosporin therapy has proven it to be a highly effective agent in the treatment of psoriasis; which provides rapid and sustained disease remission, and significantly improves patient QoL. The 
side-effect profile is well known and predictable, and adherence to treatment guidelines extensively reduces the risk of adverse events. The use of intermittent shortcourse ciclosporin therapy has been established as the optimum treatment regimen for most patients, although long-term continuous therapy remains necessary in a small subgroup with refractory disease.

The recommendations in this consensus statement provide guidance for any clinician who is either considering the use of, or continuing to use, ciclosporin in the management of psoriasis.

\section{Acknowledgments}

The members of the 'Ciclosporin in psoriasis clinical practice' consensus committee include: Professor Christopher Griffiths (chairman), Dermatology Centre, University of Manchester, Hope Hospital, Manchester, UK; Professor Louis Dubertret, Skin Research Institute and Dermatology, Saint Louis University Hospital, Paris, France; Professor Charles Ellis, Department of Dermatology, University of Michigan Medical School, Ann Arbor, Michigan, USA; Professor Andrew Y. Finlay, Department of Dermatology, University of Wales College of Medicine, Cardiff, UK; Professor Aldo Finzi, Department of Dermatology, University of Milan, Milan, Italy; Professor Vincent Ho, Division of Dermatology, University of British Columbia, Vancouver, British Columbia, Canada; Dr Atholl Johnston, Department of Clinical Pharmacology, Barts and The London, University of London, UK; Professor Andreas Katsambas, Department of Dermatology, University of Athens, A.Sygros Hospital, Athens, Greece; Professor Arno-E. Lison, Department of Nephrology \& Transplantation, Medizinische Klinik III, Zentralkrankenhaus, Bremen, Germany; Professor Jean-Marie Naeyaert, Department of Dermatology, Ghent University Hospital, Gent, Belgium; Professor Hidemi Nakagawa, Department of Dermatology, Jichi Medical School, Tochigi, Japan; Dr Carle Paul, Clinical Research Department, Novartis Pharma AG, Basel, Switzerland, and Department of Dermatology, Mulhouse General Hospital, Mulhouse, France; and Dr Francisco Vanaclocha, Department of Dermatology, Hospital 12 de Octubre, Madrid, Spain.

Christopher Griffiths has been a funded speaker and consultant for Novartis Pharmaceuticals. Charles Ellis has worked as a consultant for Novartis Pharmaceuticals. Andrew Finlay is a member of the UK Novartis Dermatology Advisory Board. Vincent Ho has been a funded speaker for Novartis Pharmaceuticals. Atholl
Johnston has worked as a consultant for Novartis Pharmaceuticals. Jean-Marie Naeyaert has been a funded speaker for Novartis Pharmaceuticals. Carle Paul is currently an employee of Novartis Pharmaceuticals.

\section{References}

1 Griffiths CEM, Clark CM, Chalmers RJG, Li Wan Po A, Williams HC. A systematic review of treatments for severe psoriasis. Health Technol Assess 2000; 4: 40.

2 Berth-Jones J, Voorhees JJ. Consensus conference on cyclosporin A microemulsion for psoriasis, June 1996. Br J Dermatol 1996; 135: $775-7$.

3 Kirby B, Fortune DG, Bhushan M, Chalmers RJ, Griffiths CEM. The Salford Psoriasis Index: an holistic measure of psoriasis severity. Br J Dermatol 2000; 142: 728-32.

4 Koo J, Kozma CM, Reinke K. The development of a disease-specific questionnaire to assess quality of life for psoriasis patients: an analysis of the reliability, validity, and responsiveness of the Psoriasis Quality of Life Questionnaire. Dermatol Psychosom 2002; 3: 171-9.

5 Zachariae R, Zachariae H, Blomqvist K et al. Quality of life in 6497 Nordic patients with psoriasis. Br J Dermatol 2002 June; 146: $1006-16$.

6 Hermansen SE, Helland CA, Finlay AY. Patients' and doctors' assessment of skin disease handicap. Clin Exp Dermatol 2002; 27: 249-50.

7 Vardy D, Besser A, Amir M, Gesthalter B, Biton A, Buskila D. Experiences of stigmatization play a role in mediating the impact of disease severity on quality of life in psoriasis patients. $\mathrm{Br} J$ Dermatol 2002; 147: 736-42.

8 Renzi C, Picardi A, Abeni D et al. Association of dissatisfaction with care and psychiatric morbidity with poor treatment compliance. Arch Dermatol 2002; 138: 337-42.

9 Richards HL, Fortune DG, O'Sullivan TM, Main CJ, Griffiths CEM. Patients with psoriasis and their compliance with medication. J Am Acad Dermatol 1999; 41: 581-3.

10 Fortune DG, Richards HL, Kirby B et al. Psychological distress impairs clearance of psoriasis in patients treated with photochemotherapy. Arch Dermatol 2003; 139: 752-6.

11 Krueger GG, Feldman SR, Camisa C et al. Two considerations for patients with psoriasis and their clinicians: what defines mild, moderate, and severe psoriasis? What constitutes a clinically significant improvement when treating psoriasis? J Am Acad Dermatol 2000; 43: 281-5.

12 Kirby B, Richards HL, Woo P, Hindle E, Main CJ, Griffiths CEM. Physical and psychologic measures are necessary to assess overall psoriasis severity. J Am Acad Dermatol 2001; 45: 72-6.

13 Krueger G, Koo J, Lebwohl M, Menter A, Stern RS, Rolstad T. The impact of psoriasis on quality of life: results of a 1998 National Psoriasis Foundation patient-membership survey. Arch Dermatol 2001; 137: 280-4.

14 Ellis CN, Fradin MS, Messana JM et al. Cyclosporine for plaquetype psoriasis: results of a multi-dose, double-blind trial. $N$ Engl J Med 1991; 324: 277-84.

15 Finzi AF, ed. Cyclosporin in severe psoriasis: The Italian experience. Dermatology, 1993: 187 (Suppl. 1).

16 Berth-Jones J, Henderson CA, Munro CS et al. Treatment of psoriasis with intermittent short-course cyclosporin 
$\left(\right.$ Neoral $\left.^{\circledR}\right)$. A multicentre study. Br J Dermatol 1997; 136: 527-30.

17 Ho VCY, Albrecht G, Vanaclocha F et al. Intermittent short courses of cyclosporin $\left(\mathrm{Neoral}^{\circledR}\right.$ ) for psoriasis unresponsive to topical therapy: a 1-year multicentre, randomised study. $\mathrm{Br} \mathrm{J}$ Dermatol 1999; 141: 283-91.

18 Ho VCY, Griffiths CEM, Berth-Jones J et al. Intermittent short courses of cyclosporine microemulsion for the long-term management of psoriasis: a 2-year cohort study. J Am Acad Dermatol 2001; 44: 643-51.

19 Faerber L, Braeutigam M, Weidinger G et al. Cyclosporine in severe psoriasis: Results of a meta-analysis. Am J Clin Dermatol 2001; 2: 41-7.

20 Touw CR, Hakkaart-Van Roijen L, Paul C, Rutten EFH, Finlay AY. Quality of life and clinical outcome in psoriasis patients using intermittent cyclosporin. $\mathrm{Br} J$ Dermatol 2001; 144: 967-72.

21 Shupack J, Abel E, Bauer E et al. Cyclosporine as maintenance therapy in patients with severe psoriasis. J Am Acad Dermatol 1997; 36: 423-32.

22 Koo J, OLP302 Study Group. A randomised, double-blind study comparing the efficacy, safety and optimal dose of two formulations of cyclosporin, Neoral and Sandimmun, in patients with severe psoriasis. Br J Dermatol 1998; 139: 88-95.

23 Powles AV, Baker BS, Valdimarsson H, Hulme B, Fry L. Four years of experience with cyclosporin A for psoriasis. Br J Dermatol 1990; 36 (Suppl.): 13-9.

24 Ohtsuki M, Nakagawa H, Sugai J et al. Long-term continuous versus intermittent cyclosporin: therapy for psoriasis. J Dermatol 2003; 30: 290-8.

25 Mrowietz U, Faerber L, Henneicke-von Zepelin HH, Bachmann H, Welzel D, Christophers E. Long-term maintenance therapy with cyclosporine and post-treatment survey in severe psoriasis: results of a multicenter study. German Multicenter Study. J Am Acad Dermatol 1995; 33: 470-5.

26 Vanaclocha F, Puig L, Daudén E et al. Effectiveness of cyclosporine in patients with psoriasis. 9th International Psoriasis Symposium, New York, June 17-22, 2003.

27 Puig L, Vanaclocha F, Daudén E et al. Quality of life in psoriatic patients treated with cyclosporine. 9th International Psoriasis Symposium, New York, June 17-22, 2003.

28 Mihatsch MJ, Belghiti D, Bohman SO et al. Kidney biopsies in control or cyclosporin A treated psoriatic patients. Br J Dermatol 1990; 122: 95-100.

29 Lowe NJ, Wieder JM, Rosenbach A et al. Long-term low-dose cyclosporine therapy for severe psoriasis: effects on renal function and structure. J Am Acad Dermatol 1997; 37: 671-2.

30 Mihatsch MJ, Wolff K. A consensus report: cyclosporin A therapy for psoriasis. Br J Dermatol 1990; 122 (Suppl. 26): 1-3.

31 Feutren G, Mihatsch MJ. Risk factors for cyclosporin-induced nephropathy in patients with autoimmune diseases. International Kidney Biopsy Registry of Cyclosporin in Autoimmune Diseases. N Engl J Med 1992; 326: 1654-60.

32 Grossman RM, Chevret S, Abi-Rached J et al. Long-term safety of cyclosporin in the treatment of psoriasis. Arch Dermatol 1996; 132: 623-9.

33 Kaoukhov A, Paul C, Lahfa M, Blanchet F, Dubertret L. Renal function during long-term cyclosporin treatment of psoriasis. Ann Dermatol Venereol 2000; 127: 180-3.

34 Laburte C, Grossman R, Abi-Rached J, Abeywickrama KH, Dubertret L. Efficacy and safety of oral cyclosporin A (CyA; Sandimmun) for long-term treatment of chronic severe plaque psoriasis. Br J Dermatol 1994; 130: 366-75.
35 Young EW, Ellis CN, Messana JM et al. A prospective study of renal structure and function in patients treated with cyclosporin. Kidney Internat 1994; 46: 1216-22.

36 Paul CF, Ho VC, McGeown C et al. Risk of malignancies in psoriasis treated with cyclosporine: a 5-yr cohort study. J Invest Dermatol 2003; 120: 211-6.

37 Marcil I, Stern RS. Squamous-cell cancer of the skin in patients given PUVA and ciclosporin: nested cohort crossover study. Lancet 2001; 358: 1042-5.

38 Cockburn ITR, Krupp P. The risk of neoplasms in patients treated with cyclosporin A. J Autoimmun 1989; 2: 723-31.

39 Jonas S, Rayes N, Neumann U et al. De novo malignancies after liver transplantation using tacrolimus-based protocols or cyclosporin-based quadruple immunosuppression with an interleukin-2 receptor antibody or anti-thymocyte globulin. Cancer 1997; 80: 1141-50.

40 Frentz G, Olsen JH. Malignant tumours and psoriasis: a follow-up study. Br J Dermatol 1999; 140: 237-42.

41 Gelfand JM, Berlin J, Van Voorhees A, Margolis DJ. Lymphoma rates are low but increased in patients with psoriasis: results from a population-based cohort study in the United Kingdom. Arch Dermatol 2003; 139: 1425-9.

42 Margolis D, Biker W, Hennessy S, Vittorio C, Santanna J, Strom BL. The risk of malignancy associated with psoriasis. Arch Dermatol 2001; 137: 778-83.

43 Stern RS, Lunder EJ. Risk of squamous cell carcinoma and methoxsalen (psoralens) and UV-A radiation (PUVA); a metaanalysis. Arch Dermatol 1998; 134: 1582-5.

44 Kreimer-Erlacher H, Seidl H, Back B, Cerroni L, Kerl H, Wolf P. High frequency of ultraviolet mutations at the INK4a-ARF locus in squamous cell carcinomas from psoralen-plus-ultraviolet-Atreated psoriasis patients. J Invest Dermatol 2003 April; 120: 676-82.

45 Nijsten TE, Stern RS. The increased risk of skin cancer is persistent after discontinuation of psoralen+ultraviolet A: a cohort study. J Invest Dermatol 2003; 121: 252-8.

46 Nijsten TE, Stern RS. Oral retinoid use reduces cutaneous squamous cell carcinoma risk in patients with psoriasis treated with psoralen-UVA: a nested cohort study. J Am Acad Dermatol 2003; 49: $644-50$.

47 Katz HI. Potential drug interactions with cyclosporine. Int J Dermatol 1997; 36 (Suppl. 1): 18-24.

48 Guidelines Subcommittee 1999 World Health OrganizationInternational Society of Hypertension. Guidelines for the Management of Hypertension. J Hypertens 1999; 17: 151-83.

492003 European Society of Hypertension. European Society of Cardiology guidelines for the management of arterial hypertension. J Hypertens 2003; 21: 1011-53.

50 Joint National Committee on Prevention, Detection, Evaluation and Treatment of High Blood Pressure. Seventh Report of the Joint National Committee on Prevention, Detection, Evaluation, and Treatment of High Blood Pressure. JAMA 2003; 289: 256072 .

51 Midtvedt K, Fauchald P, Bergan S et al. $\mathrm{C}_{2}$ monitoring in maintenance renal transplant recipients: is it worthwhile? Transplantation 2003; 76: 1236-8.

52 Cockcroft DW, Goult MH. Prediction of creatinine clearance from serum creatinine. Nephron 1976; 16 (1): 31-41.

53 Feutren G, Friend D, Timonen P, Barnes A, Laburte C. Predictive value of cyclosporin A level for efficacy or renal dysfunction in psoriasis. Br J Dermatol 1990; 122 (Suppl. 36): 85-93.

54 Heydendael VM, Spuls PI, Ten Berge IJ, Opmeer BC, Bos JD, de Rie MA. Cyclosporin trough levels: is monitoring necessary during 
short-term treatment in psoriasis? A systematic review and clinical data on trough levels. Br J Dermatol 2002; 147: 122-9.

55 Clark CM, Kirby B, Morris AD et al. Combination treatment with methotrexate and cyclosporin for severe recalcitrant psoriasis. $\mathrm{Br}$ J Dermatol 1999; 141: 279-82.

56 Davison SC, Morris-Jones R, Powles AV, Fry L. Change of treatment from cyclosporin to mycophenolate mofetil in severe psoriasis. Br J Dermatol 2000; 143 (2): 405-7.

57 Ellis CN, Krueger GG for the Alefacept Clinical Study Group. Treatment of chronic plaque psoriasis by selective targeting of memory effector T lymphocytes. N Engl J Med 2001; 345: 248-55.

58 Mrowietz U. Advances in systemic therapy for psoriasis. Clin Exp Dermatol 2001; 26: 362-7.
59 Mrowietz U. Treatment targeted to cell surface epitopes. Clin Exp Dermatol 2002; 27: 591-6.

60 Johnston A, Keown PA, Holt DW. Simple bioequivalence criteria: are they relevant to critical dose drugs? Experience gained from cyclosporine. Ther Drug Monit 1997; 19: 375-81.

61 Johnston A, Holt DW. Bioequivalence criteria for cyclosporine. Transplant Proc 1999; 31: 1649-53.

62 Pollard S, Nashan B, Johnston A et al. A pharmacokinetic and clinical review of the potential clinical impact of using different formulations of cyclosporin A. Clin Ther 2003; 25: 1654-69.

63 British National Formulary. Corticosteroids and other immunosuppressants - ciclosporin. September 2003; 46: section 8.2.2 (also available at: http://www.bnf.org). 